\title{
Surgical treatment of recurrent acute lymphoblastic leukemia with humeral osteomyelitis as the first symptom: a case report and literature review
}

\author{
Yiyang Liu ${ }^{1}$, Chun Zhang ${ }^{1}$, Lifeng Shen ${ }^{2}$, Haiyong Ren ${ }^{1}$, Yangjun Lao ${ }^{1}$, Meng Zhou ${ }^{3}$, Huifang Jiang ${ }^{3}$, \\ Bingyuan Lin ${ }^{1}$ \\ ${ }^{1}$ Department of Orthopedics, Tongde Hospital of Zhejiang Province, Hangzhou, China; ${ }^{2}$ Department of Orthopedics, Sir Run Run Shaw Hospital of \\ Zhejiang University School of Medicine, Hangzhou, China; ${ }^{3}$ Department of Hematology, Tongde Hospital of Zhejiang Province, Hangzhou, China \\ Correspondence to: Bingyuan Lin, MD. Department of Orthopedics, Tongde Hospital of Zhejiang Province, 234 Gucui Rd., Hangzhou, China. \\ Email: hzboneage@163.com.
}

\begin{abstract}
Osteomyelitis is a complication of acute lymphoblastic leukemia (ALL); however, its incidence rate is low. Recurrent ALL for which osteomyelitis is the first manifestation is rarer still. The repair and reconstruction of segmental bone defects caused by infection is a clinical problem. This article reports a rare case study of a 22-year-old male patient with recurrent ALL for which right humeral osteomyelitis was the first symptom including chills at first, recurrent fever and chills then, purulence from poster lateral right upper arm finally. Before treatment, the patient concealed his previous history of ALL. Debridement was performed, and the Masquelet technique and free fibula transplantation were used to reconstruct the humerus. ALL recurred with extensive bone invasion throughout the body 10 weeks after the humeral reconstruction. Despite the success of bone marrow transplantation, the patient died of complications. A limited follow-up period reveled that while leukemia extensively affected the bones of the patient's body, the reconstruction of the humerus was successful, and bone healing was good. This case study suggests that atypical or unexplained osteoarticular infection may be the precursor to some systemic diseases, and that Masquelet technology combined with free fibula transplantation is one of the best choices to treat segmental bone defects.
\end{abstract}

Keywords: Osteomyelitis; acute lymphoblastic leukemia (ALL); orthopedic surgery; Fibular transplantation; Masquelet technology

Submitted Feb 04, 2021. Accepted for publication Apr 01, 2021.

doi: $10.21037 / \mathrm{atm}-21-1112$

View this article at: http://dx.doi.org/10.21037/atm-21-1112

\section{Introduction}

Very few patients with acute lymphoblastic leukemia (ALL) experience complications with osteomyelitis during chemotherapy (1-6). Recurrent ALL for which the first symptom is osteomyelitis involving the entire long bone is very rare. The resulting long segmental bone defect is difficult to treat in clinical orthopedics. Whether the Ilizarov technique, the Masquelet technique, or a simple free fibula transplantation is used, the comprehensive effect is not satisfactory. This article reports a case study of a patient with recurrent ALL for whom the first symptom was right humeral osteomyelitis. After thorough debridement, Masquelet technology combined with free fibula transplantation was used to reconstruct the patient's humerus, and satisfactory results were achieved.

We present the following article in accordance with the CARE reporting checklist (available at http://dx.doi. org/10.21037/atm-21-1112). 

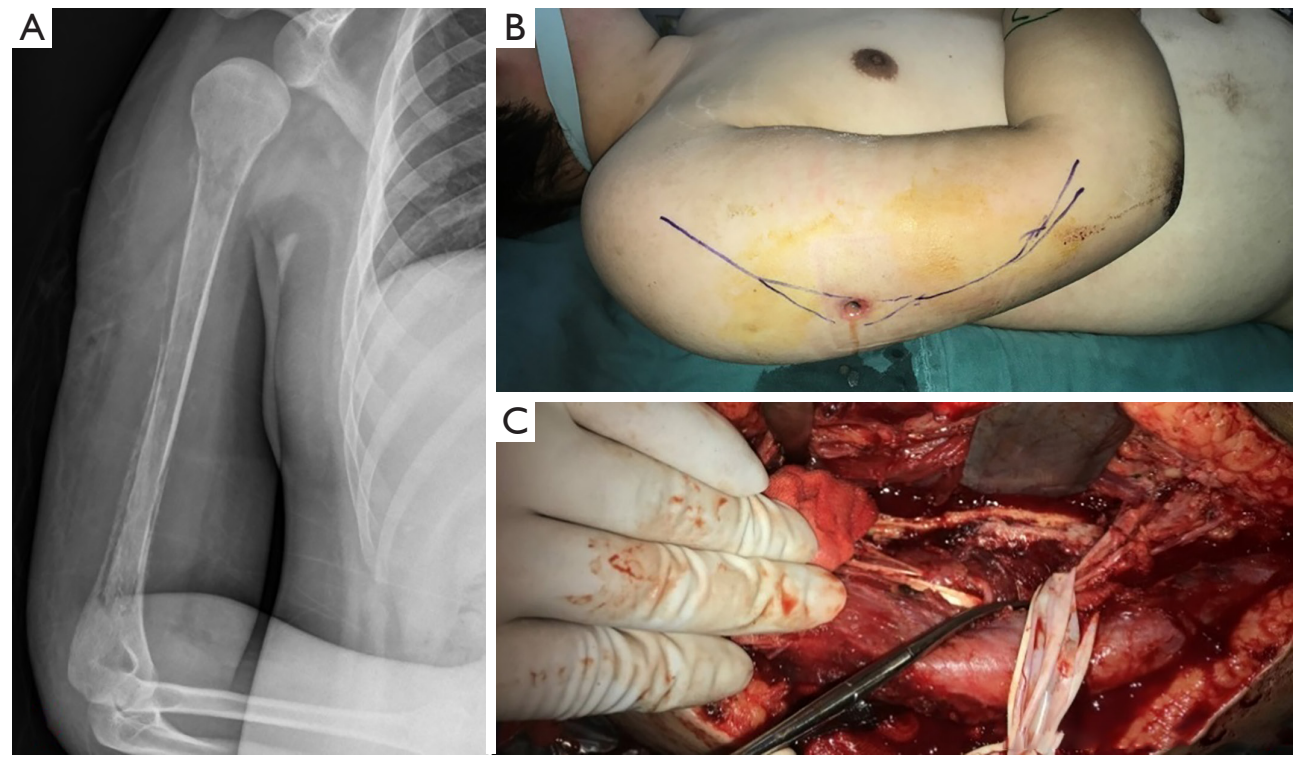

Figure 1 Preoperative images and first intraoperative photos. Before the first operation, an X-ray (A) showed the uneven bone density of the right humerus shaft, the thin cortex, the visible callus formation, and the pathological fractures of the distal and proximal humerus shaft. During the operation, the posterolateral sinus tract of the right upper arm was visible (B), the whole humerus was immersed in pus, and the medullary cavity was filled with purulent tissue (C).

\section{Case presentation}

A 22-year-old male felt pain in his right upper arm after lifting dumbbells on November 20, 2017, and reported experiencing chills that night. He felt feverish but did not take his temperature. The next day, the patient did not experience chills, but he reported that, despite no obvious redness or local swelling, his right upper arm felt uncomfortable for the next 3 weeks. After 9 days of acupuncture and moxibustion treatment since December 10th, the pain in the patient's arm was relieved, but he regularly experienced "fever in the morning, chills in the daytime, chills at sunset, and body heat at night"; no measure of his body temperature was taken. On the night of December 24, 2017, the posterolateral rupture of the right upper arm was purulent, and the patient was hospitalized the next day, at which time the patient concealed his chemotherapy history of ALL 3 years ago, but revealed his history of appendicitis surgery. On the night of his admission, the patient's body temperature was $39.1{ }^{\circ} \mathrm{C}$, he had a c-reactive protein (CRP) of $76.78 \mathrm{mg} / \mathrm{L}$, and his total number of white blood cells (WBCs) and various classifications were within the normal range. A culture of secretory bacteria suggested salmonella enteritidis. His diagnosis at admission was chronic osteomyelitis of right humerus.

On January 2, 2018, the patient was placed under general anesthesia and underwent the "external fixation of right humeral fracture, debridement of osteomyelitis, implantation of vancomycin and gentamicin calcium sulfate, vacuum sealing and drainage" (see Figure 1). One week later (on January 9, 2018), "right humeral osteomyelitis debridement, loading vancomycin and gentamicin bone cement open placement" were performed (see Figure 2). The samples from both operations indicated "inflammatory lesions," but the bacterial culture was negative. Imipenem combined with rifampicin were used for anti-infection. On April 9, 2018, when the induction membrane around the bone cement rod was fully formed, "right humerus bone cement removal, free fibula transplantation, autologous iliac bone mixed with vancomycin and gentamicin calcium sulfate bone grafting" were performed (see Figure 3). During the operation, part of the induced membrane was removed for examination. The bacterial culture was negative, and the disease was reported as an "infiltration of hyperplastic fibrous tissue, small focal lymphocytes and plasma cells." After the operation, the patient was treated with linezolid, and a combination of linezolid, and rifampin for 8 weeks.

10 weeks after the fibula transplantation, the patient 

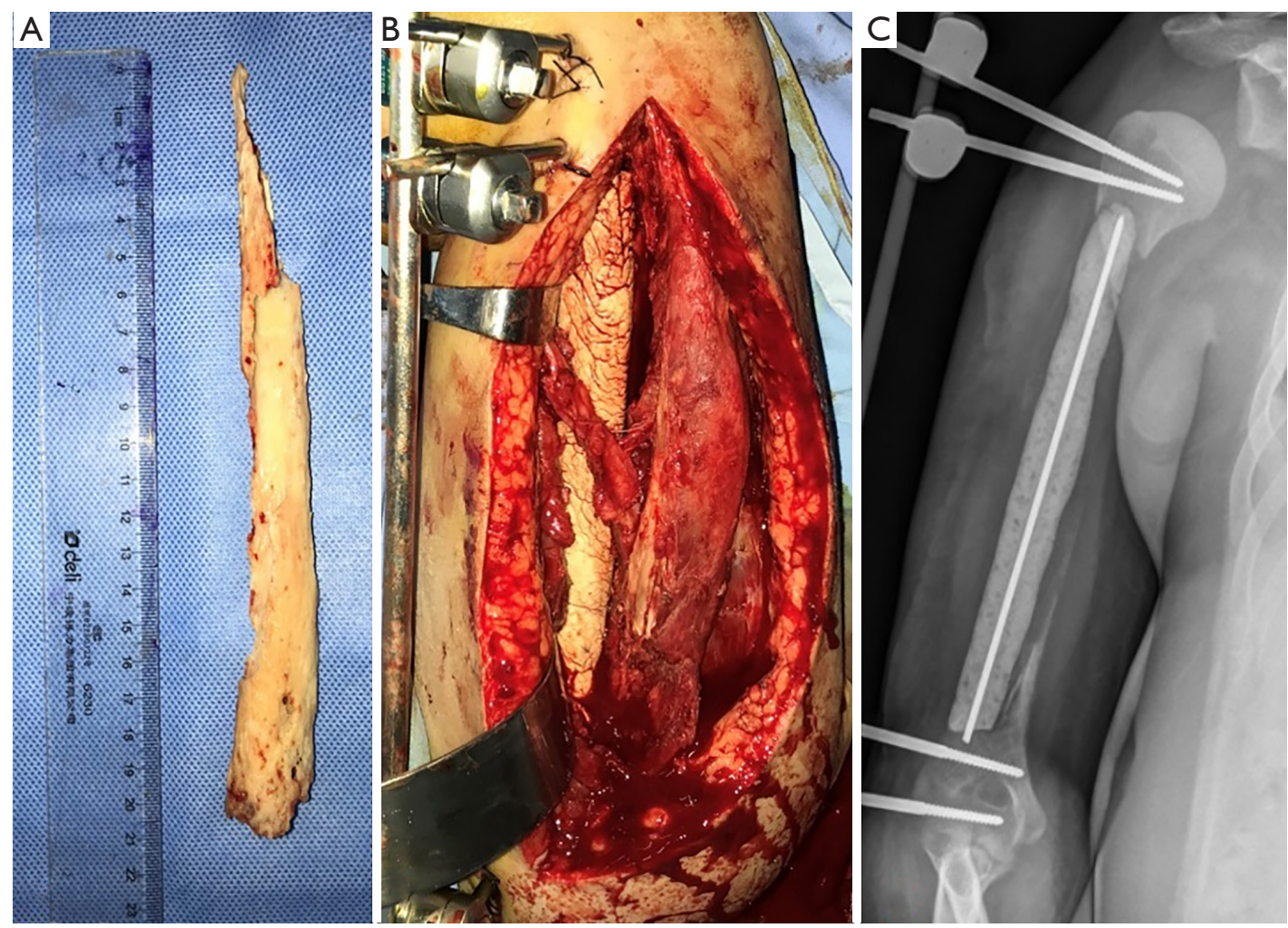

Figure 2 Photos from the second operation. In the second operation on January 9, 2018, the entire $21 \mathrm{~cm}$ long humeral shaft lesion was taken out of the dead bone (A), and a prefabricated antibiotic bone cement rod was implanted in the humeral shaft bone lesion to occupy the place (B); a postoperative X-ray showed that the bone cement and the humeral force line was good. To strengthen the local mechanical strength, $2.5 \mathrm{~mm}$ K-wire was inserted into the antibiotic bone cement rod to achieve the effect of "reinforced concrete" (C).

reported feeling pain in his left shoulder. Imaging revealed no obvious abnormalities, but routine blood testing showed a significant increase in the total number of WBCs and lymphocytes. After a hematology consultation, a diagnosis of ALL was suggested for the first time. The patient insisted that he be discharged to another hospital for treatment; however, ultimately, he did not seek further treatment. The patient was hospitalized again because of systemic pain on August 30, 2018, and presented with extensive skeletal destruction (see Figure 4). At this time, the patient revealed that he had undergone one course of chemotherapy to treat ALL 3 years ago, and that this caused appendiceal perforation and peritonitis. Postoperatively, the patient refused any treatment. He was admitted to the hematology department on the same day and received chemotherapy. He underwent a successful bone marrow transplantation on February 27, 2019. However, due to pulmonary infection, the patient died on November 21, 2019.

During the limited follow-up period, no signs of local infection occurred in the right upper arm. 14 months after the operation, in the case of extensive bone destruction throughout the body, a ring-shaped callus had formed around the fibula flap, which fully healed the bone (see Figure 4).

All procedures performed in studies involving human participants were in accordance with the ethical standards of the institutional and/or national research committee(s) and with the Helsinki Declaration (as revised in 2013). Written informed consent was obtained from the patient.

\section{Discussion}

The patient's morbidity and pathological characteristics had the basic characteristics of osteomyelitis, but the clinical manifestations differed to those of typical blood-borne osteomyelitis. First, local infection was serious, and while systemic symptoms were serious, local symptoms were light. The patient's CRP was high, but the total number 

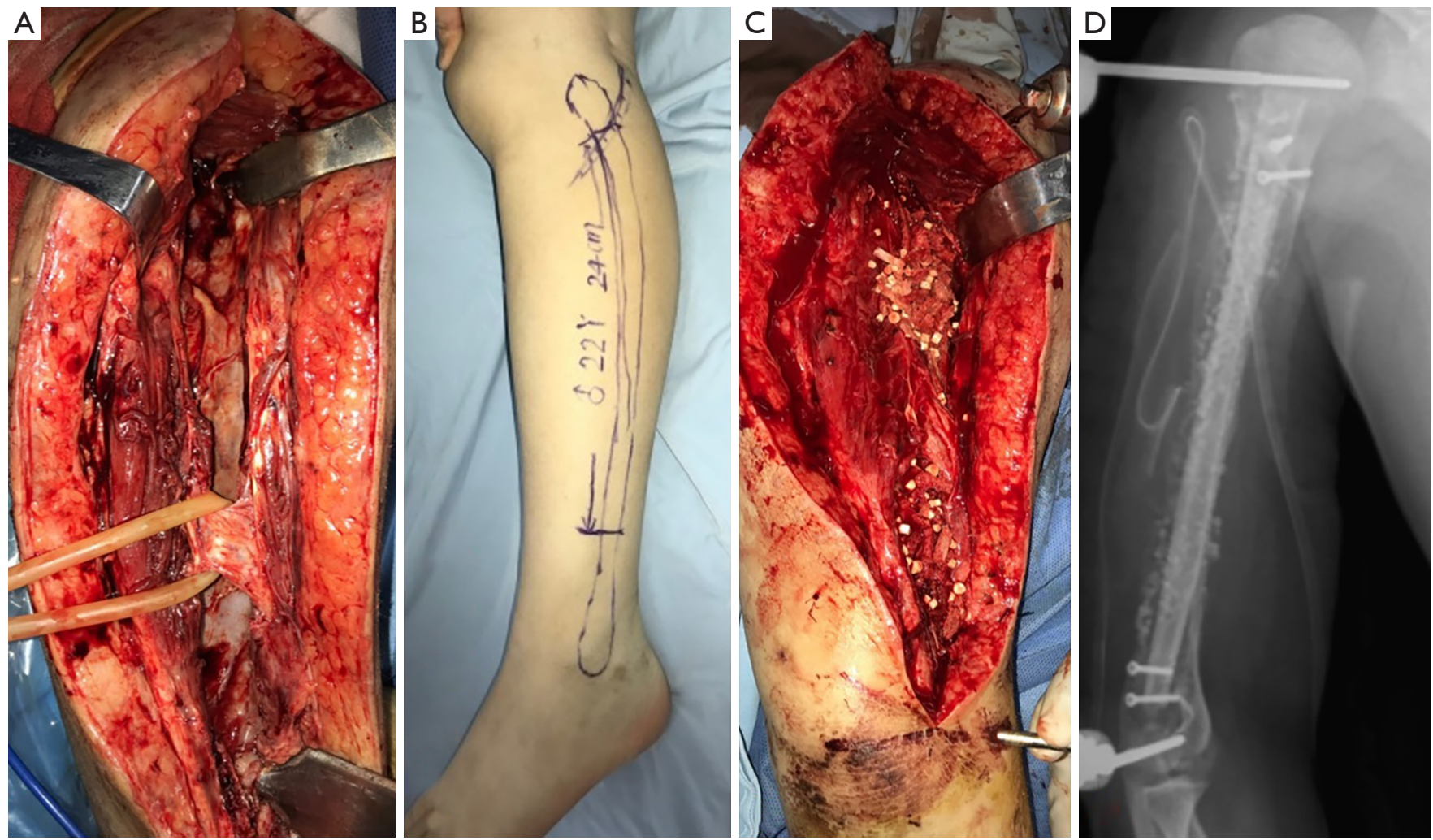

Figure 3 Photos from the third operation. In the third operation on April 9, 2018, the bone cement rod was removed during the operation, and the local induced membrane was fully formed (A). The design of the fibula flap on the left calf (B). The implanted area was implanted with limited internal fixation with screws. Filled with antibiotic calcium sulfate particles and autologous iliac bone around the fibula flap (C). The fibula artery and vein were anastomosed end to side, and all surgical incisions were closed. A postoperative X-ray showed that the fibula flap transplantation position and humerus force line were good, the bone graft was sufficient, and the internal and external fixation was in place (D).

of leukocytes and their classification fell within the normal range. Further, the envelope around the dead bone of the humeral shaft conformed to the general pathological characteristics of chronic osteomyelitis; however, the period from the onset of the disease to the operation was only 6 weeks, and the rapid formation and wide range of the envelope differed to that which was routine, and the surface of the dead bone was widely changed by insect erosion, and also differed to that which was routine.

Currently, there is no clear evidence as to whether this series of different manifestations was related to the patients' previous history of ALL. In the past 30 years, more than 20 patients with ALL reported osteomyelitis after undergoing chemotherapy 2-5 times (1-7). All of these patients had low-toxic bacterial infections or fungal infections $(4,5)$. Low immunological function is an important inducement of bone infection. Only two cases of ALL have been reported for which the first symptom was osteomyelitis $(8,9)$. In both these cases, the patients were children and had multiple osteomyelitis. The author of one of two case reports was of the view that one of the two children mentioned above, who presented with a low incidence of osteomyelitis, a nonspecific histopathology and laboratory tests that usually showed negative bacterial cultures, had chronic recurrent multifocal osteomyelitis (CRMO) $(10,11)$. This child's case was similar to the one in the present case study.

After debridement, a bone cement rod was used to fill the humerus shaft bone defect for the patient in the present case study. Due to the large range of bone defects, a $2.5-\mathrm{mm}$ diameter Kirschner wire was placed in the center of the prefabricated bone cement rod to form a "reinforced concrete" mode to strengthen the local mechanical strength during bone cement openness. Notably, no research appears to have been conducted on the "bone cement-Kirschner 

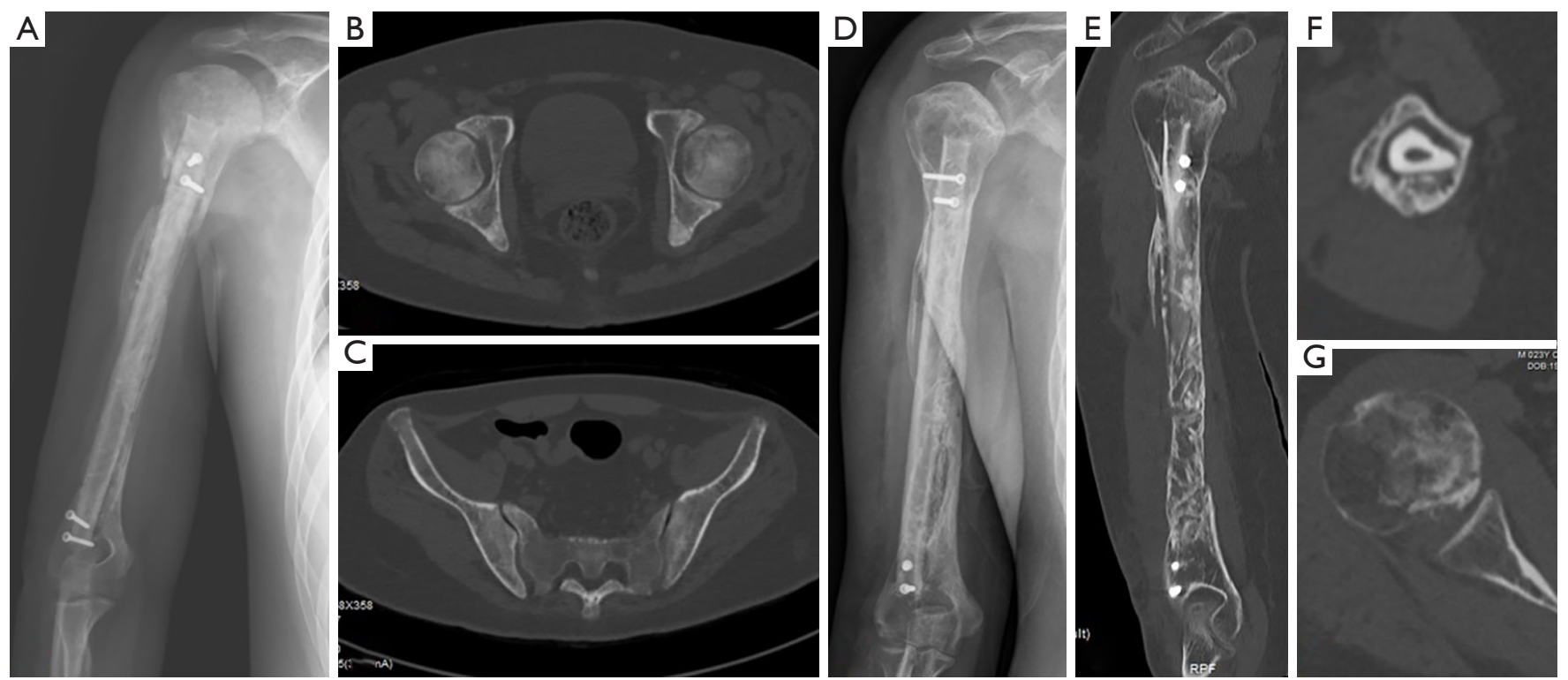

Figure 4 Imaging after 20 weeks of reconstruction of humerus. 20 weeks after fibula transplantation (August 30, 2018), an X-ray showed that the osteogenesis around the fibula flap was good, but a low-density shadow-like insect erosion could be seen in the humeral head (A); computed tomography (CT) showed the femoral head and acetabulum (B), the sacrum and ilium (C) bone destruction to varying degrees. 14 months after the fibula transplantation, an X-ray (D) and a CT reconstruction (E) showed that the fibula flap had thickened significantly and was surrounded by an obvious continuous callus; a CT plain scan (F) showed that a good circular callus had been formed around the fibula flap; however, the bone density of the humeral head remained low and there was significant bone destruction (G).

wire" model to date, and its mechanical properties require further study.

Autogenous cancellous bone grafting is the "gold standard" of the classic Masquelet technique for secondstage bone grafting. However, for the patient in the present case study, a pure autologous cancellous bone graft did not effectively fill the cavity, or provide mechanical support and stability. A simple fibula transplantation can cause bone healing and restore the length of the limbs; however, as the fibula and humerus are very different in diameter, the local mechanical strength after transplantation cannot fulfil patients' normal physiological needs. Thus, a vascularized free fibula transplantation combined with autogenous cancellous bone and mixed bone grafts loaded with vancomycin and gentamicin calcium sulfate were used. This approach was adopted for the following reasons: (I) segmental bone transplantation of the fibula can be used to reconstruct bone continuity and provide mechanical support; (II) after the whole fibula is grafted, it effectively takes up space in the cavity formed by the induced membrane, which greatly reduces the amount of autogenous cancellous bone; (III) grafting of the fibular flap with microvascular anastomosis, improves the success rate of bone transplantation and is conducive to bone healing; (IV) compared with simple free fibula transplantation, this surgical method is beneficial to local osteogenesis, and increases the circumference of the fibula flap; and (V) the degradable loaded vancomycin and gentamicin calcium sulfate granules can enable highly concentrated local-antibiotic delivery and prevent the need for a second operation.

This patient's bone transplantation resulted in satisfactory bone healing; however, it should be noted there was extensive bone destruction in the whole body due to osteogenesis. Previous studies of patients with ALL have confirmed that before treatment, bone destruction is mainly caused by leukemia cells, and the lesions are more serious; however, after chemotherapy, bone destruction occurs because chemotherapy drugs inhibit bone synthesis and promote bone absorption $(2,3,9)$. After 4 months of chemotherapy, accompanied by osteoporosis and the destruction of multiple parts of the body, the patient achieved satisfactory boniness, which also confirmed that Masquelet technology combined with free fibula transplantation effectively improved the quality of bone healing. 
There is no clinical report on this operation method. However, based on the successful experience of this patient, we are of the view that this surgical treatment is an effective method for the treatment of segmental long bone defects after debridement of chronic osteomyelitis. In addition, after reviewing the course of the disease and treatment, and based on the limited literature available in this area, we suggest that (I) when a series of atypical or unexplained osteoarticular infections occur, the possibility of other systemic diseases, including tumors, should be considered; and (II) thorough debridement, effective tissue filling and an antibiotic slow-release system are key to the treatment of osteomyelitis.

\section{Acknowledgments}

Funding: Basic Public Welfare Research Project of Zhejiang Province (Grant No. GF19H060025); Natural Science Foundation of Zhejiang Province (Grant No. LY20H270002) and Traditional Chinese Medicine Science and Technology Project of Zhejiang Province (Grant No. 2021ZB060).

\section{Footnote}

Reporting Checklist: The authors have completed the CARE reporting checklist. Available at http://dx.doi.org/10.21037/ atm-21-1112

Conflicts of Interest: All authors have completed the ICMJE uniform disclosure form (available at http://dx.doi. org/10.21037/atm-21-1112). The authors have no conflicts of interest to declare.

Ethical Statement: The authors are accountable for all aspects of the work in ensuring that questions related to the accuracy or integrity of any part of the work are appropriately investigated and resolved. All procedures performed in studies involving human participants were in accordance with the ethical standards of the institutional and/or national research committee(s) and with the Helsinki Declaration (as revised in 2013). Written informed consent was obtained from the patient.

Open Access Statement: This is an Open Access article distributed in accordance with the Creative Commons
Attribution-NonCommercial-NoDerivs 4.0 International License (CC BY-NC-ND 4.0), which permits the noncommercial replication and distribution of the article with the strict proviso that no changes or edits are made and the original work is properly cited (including links to both the formal publication through the relevant DOI and the license). See: https://creativecommons.org/licenses/by-nc-nd/4.0/.

\section{References}

1. Mutoh Y, Hirai R, Tanimura A, et al. Osteomyelitis due to Clostridium innocuum in a patient with acute lymphoblastic leukemia: case report and literature review. Springerplus 2015;4:385.

2. Doganis D, Baka M, Tsolia M, et al. Multifocal Aeromonas Osteomyelitis in a Child with Leukemia. Case Rep Infect Dis 2016;2016:8159048.

3. Oh DY, Madhusoodhan PP, Springer DJ, et al. Cryptococcal osteomyelitis in an adolescent survivor of T-cell acute lymphoblastic leukemia. Pediatr Infect Dis J 2015;34:662-6.

4. Zhang WL, Shi TX, Tang CH, et al. Study on bone metabolism in children with acute lymphocytic leukemia before and after chemotherapy. J China Pediatr Blood 2004;9:204-7.

5. Liu HC, Liu YF, Li L, et al. Study on Bone Metabolism in Children with Leukemia and Its Relationship to Tumor Necrosis Factor-Alpha Pre- and Post-Treatment. J Appl Clin Pediatr 2006;21:993-4.

6. Shen Z, Zeng D, Zou Z, et al. Effects of SDF-1/CXCR4 signal pathway blockade by AMD3100 on the adhesion of leukemia cells to osteoblast niche and the drug resistance of leukemia cells. Zhonghua Xue Ye Xue Za Zhi 2015;36:413-7.

7. Wong T, Ko JY, Wang FS, et al. Epstein-Barr virus associated extranodal natural killer T cell lymphoma of nasal type mimicking pyogenic osteomyelitis of the proximal humerus. Chang Gung Med J 2008;31:314-9.

8. Li AL, Jin SH, Luo XQ. A case of acute leukemia in children with pyogenic osteomyelitis as the first symptom. J China Pediatr Blood 1999;4:31.

9. Tavil B, Secmeer G, Balc YI, et al. Chronic recurrent multifocal osteomyelitis as the first presentation of acute lymphoblastic leukemia in a 2-year-old boy. J Pediatr Hematol Oncol 2010;32:e151-e152.

10. Girschick HJ, Raab P, Surbaum S, et al. Chronic non- 
bacterial osteomyelitis in children. Ann Rheum Dis 2005;64:279-85.

11. Jurik AG. Chronic recurrent multifocal osteomyelitis.
Semin Musculoskelet Radiol 2004;8:243-53.

(English Language Editor: L. Huleatt)

Cite this article as: Liu Y, Zhang C, Shen L, Ren H, Lao Y, Zhou M, Jiang H, Lin B. Surgical treatment of recurrent acute lymphoblastic leukemia with humeral osteomyelitis as the first symptom: a case report and literature review. Ann Transl Med 2021;9(8):722. doi: 10.21037/atm-21-1112 